\title{
Gestion et ingénierie des systèmes hospitaliers à l'ère du numérique et des innovations organisationnelles
}

\section{Management and engineering of hospital systems in the digital age and organizational innovations}

\author{
Sondes Chaabane ${ }^{1,2}$, Maria Di Mascolo ${ }^{3}$, Franck Fontanili ${ }^{4}$ \\ ${ }^{1}$ Univ. Polytechnique Hauts-de-France, LAMIH, CNRS, UMR 8201, F-59313 Valenciennes, France \\ ${ }^{2}$ INSA Hauts-de-France, F-59313 Valenciennes, France sondes.chaabane@uphf.fr \\ ${ }^{3}$ Univ. Grenoble Alpes, CNRS, Grenoble INP, G-SCOP, 38000 Grenoble, France maria.di-mascolo@grenoble-inp.fr \\ ${ }^{4}$ IMT - Albi, Centre de Génie Industriel, 81013 Albi, France franck.fontanili@mines-albi.fr
}

\section{Editorial}

Les établissements de soins et de santé évoluent de plus en plus vers des établissements agiles, performants et ouverts sur leurs environnements. Les avancées technologiques et les innovations organisationnelles couplées avec les méthodes et les outils du génie industriel contribuent à ce que les établissements puissent maîtriser et optimiser l'offre de soins, anticiper et prévenir la demande et contrôler l'évolution des besoins en soins et santé. L'humain prend de plus en plus une place au centre des préoccupations en sa qualité de patient, citoyen et soignant. L'objectif est d'offrir un environnement de santé performant répondant aux exigences de sécurité, de qualité et de productivité.

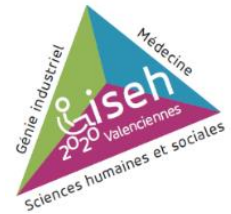

Ce numéro spécial fait suite à la $10^{\text {ème }}$ Conférence Francophone en Gestion et Ingénierie des Systèmes Hospitaliers - GISEH 2020 - qui a eu lieu à Valenciennes et en ligne en Octobre 2020.

Six articles ont été soumis à ce numéro spécial. Après un examen rigoureux, réalisé par plusieurs relecteurs anonymes, que nous remercions pour leur précieuse contribution, les 3 articles qui suivent ont été acceptés. Ils proposent des innovations organisationnelles et des solutions basées sur des techniques et des méthodes utilisées en génie industriel.

1. L'article de Estelle Bretagne, Jérémy Fonséca, et Eric Serra, intitulé «Application de la robotique humanö̈de au traitement de la douleur », s'intéresse à la question suivante : Comment, à l'ère du numérique, un robot humanoïde peut-il aider à améliorer la qualité de vie des citoyens douloureux ? Il montre l'intérêt d'utiliser un robot humanoïde pour interagir avec des patients douloureux (permet de fournir des informations au patient, permet une évaluation et un suivi de la douleur, détourne l'attention, propose des réponses thérapeutiques adaptées et sélectionnées au préalable par le patient et son médecin). Il étudie ensuite la collaboration entre l'homme et un robot humanoïde d'assistance en améliorant l'évaluation de l'expérience douloureuse par le robot grâce à divers capteurs, dans le but de fournir des 
solutions qui répondent aux besoins réels du patient et des professionnels de la santé dans un contexte médical, et non pas aux avancées technologiques en robotique.

2. L'article de Anne Quesnel-Barbet, Julien Soula, Erik-André Sauleau, Pierre Bazile, François Dufossez, Gilles Maignant, Frédéric Albert, Arnaud Hansske, et Lorraine Trilling, intitulé «PoleSat, modélisation de la planification sanitaire pour l'aide à la décision. Application à la réorganisation de l'offre de cathétérisme vasculaire au niveau géographique des groupements hospitaliers de territoire ", s'intéresse à l'optimisation annuelle de l'Hôpital public, dans un contexte de réorganisation territoriale, d'évolution rapide des technologies médicales et d'équilibre budgétaire. Il présente le contexte de la réorganisation hospitalière, les enjeux et la problématique scientifique qui en découle, puis les approches de modélisation existantes, avant de se consacrer à la présentation de PoleSat, un outil prospectif et stratégique de modélisation (gravitaire) et de simulation, à géométrie variable dédié à la planification sanitaire, et de l'appliquer à une activité de cathétérisme vasculaire du territoire GHT Atlantique 17, regroupant plusieurs centres hospitaliers. La simulation de plusieurs scenarii montre l'utilité et la robustesse de PoleSat et met en évidence de possibles évolutions techniques, afin de rendre l'outil PoleSat plus facilement transposable à la vie réelle, mais aussi de l'étendre à d'autres domaines d'application que celui de la santé.

3. L'article de Alois Franzino, Lorraine Trilling, Maria Di Mascolo, et Thibaud Monteiro, intitulé "Intérêt de la mutualisation des professionnels pour la planification d'activités médico-sociales » s'intéresse à l'organisation d'activités de soins à domicile, et plus particulièrement la planification des accompagnements d'un ensemble d'enfants en situation de handicap par un SESSAD (Service d'Education Spéciale et de Soins A Domicile). Cette planification implique de décider conjointement de l'horaire de chaque prise en charge et du trajet des soignants. Pour organiser cette planification, il faut déterminer le professionnel en charge de chaque acte, où celui-ci se déroulera et comment organiser le planning pour réduire les frais de déplacement tout en maximisant la satisfaction des personnes recevant les soins. Pour apporter une aide aux décideurs, cet article propose un modèle de programmation linéaire en nombres entiers (PLNE) qui permet, par une approche lexicographique, d'optimiser le temps de déplacement des professionnels, tout en gardant un temps d'attente réduit. L'analyse d'une étude de cas permet d'étudier les besoins réels ainsi que les solutions qui peuvent être apportées et valide l'intérêt de mutualiser les professionnels pour planifier au mieux leurs tournées. 